\title{
Chiral Lagrangians from lattice gauge theories in the strong coupling limit
}

\author{
Taro Nagao \\ Department of Physics, Graduate School of Science, Osaka University, Toyonaka, Osaka 560-0043, Japan \\ Shinsuke M. Nishigaki* \\ Department of Physics 83 Astronomy, State University of New York, Stony Brook, NY 11794-3800, USA and \\ Department of Physics, Tokyo Institute of Technology, O-okayama, Meguro, Tokyo 152-8551, Japan
}

(December 29, 2000)

\begin{abstract}
We derive nonlinear $\sigma$ models (chiral Lagrangians) over symmetric spaces $\mathrm{U}(n), \mathrm{U}(2 n) / \operatorname{Sp}(2 n)$, and $\mathrm{U}(2 n) / \mathrm{O}(2 n)$ from $\mathrm{U}(N), \mathrm{O}(N)$, and $\mathrm{Sp}(2 N)$ lattice gauge theories coupled to $n$ flavors of staggered fermions, in the large- $N$ and $g^{2} N$ limit. To this end, we employ Zirnbauer's color-flavor transformation. We prove the spatial homogeneity of the vacuum configurations of mesons by explicitly solving the large- $N$ saddle point equations, and thus establish the above patterns of spontaneous chiral symmetry breaking without any assumptions.
\end{abstract}

PACS number(s): 11.15.Ha, 11.15.Me, 11.15.Pg, 11.30.Rd

Introduction. The concept of spontaneous symmetry breaking introduces an essential parallelism between the spectral statistics of disordered condensate [1] and the low-energy dynamics of strongly coupled gauge field theories [2], involving a juxtaposition of the lightest and weakly coupled Goldstone particles in these theories: diffusion modes associated with spontaneous breakdown of the symmetry between advanced and retarded Green's functions, and pions associated with spontaneous breakdown of the chiral symmetry. However there is a considerable difference at the practical level. An ensemble averaging can be analytically performed for disordered Hamiltonians, so that the Goldstone manifold and the nonlinear $\sigma$ model over it describing the low-frequency regime of the theory manifest themselves without ambiguity. On the other hand, gauge theories with propagating gluons do not admit such an analytic computation. Accordingly, in order to identify a low-energy effective theory one needs to appeal to nonperturbative theorems, namely Vafa-Witten's theorem [3] that forbids spontaneous breaking of the vector symmetry in a vectorial theory, and 't Hooft's anomaly matching [4] between fundamental and effective theories. Relying on these theorems, non-standard patterns of chiral symmetry breaking have been studied [5] in the context of technicolor models.

The symmetry-based approach puts a strong restriction on operators that are allowed in the effective chiral Lagrangian, but the setback is that their coupling constants are left as free parameters that can by no means be related to those of the fundamental theory. To overcome this difficulty, attempts [6] were made to derive an effective theory directly from a microscopic model under an extreme condition, namely a $\mathrm{U}(N)$ lattice gauge theory in the strong coupling limit, combined with the large- $N$ limit. This serves as a basis on which a strong coupling expansion is performed and the plaquette action can be taken into account perturbatively in $\left(g^{2} N\right)^{-1}$ [7].

Recently Altland and Simons [8] have renewed interest in this subject. Instead of computing a one-link integral via the Brézin-Gross formula [9] valid only in the large$N$ limit, they have adopted an alternative method, Zirnbauer's color-flavor transformation [10]11], which has a clear advantage of being valid at finite $N$. By converting the integrations over strongly coupled gluonic variables into supposedly weakly coupled mesonic variables, they have provided a transparent derivation of the low-energy effective action corresponding to fermions in the complex representation. However, their and all the previous works have relied on a crucial and far-from-evident assumption that the mesonic fields in the vacuum are homogeneous.

The purpose of this paper is twofold: by applying the color-flavor transformation to strongly coupled lattice gauge theories with three classical gauge groups and with staggered fermions, we shall i) demonstrate the spatial homogeneity of the mesonic fields in the vacuum, thereby establishing the chiral symmetry breaking rigorously, ii) extend the method of Altland and Simons valid for quarks in the complex representation of the gauge group, to other two classes of representations, so as to derive non-standard type of chiral Lagrangians [12].

Lattice Gauge Theory with Staggered Fermions. We consider a lattice gauge theory coupled to staggered fermions on an even $(=d)$ dimensional lattice $\mathbf{Z}^{d} \ni x$, which is split into odd and even sublattices labeled by $\circ$ and $\times$, respectively. The set of $2 d$ odd (even) sites adjacent to an even (odd) site $x$ will be denoted as $\circ_{x}\left(\times_{x}\right)$. We write the partition function with an emphasis on the bipartite nature (the lattice spacing is set to unity):

$$
\mathcal{Z}(g ; m)=\int_{G} \prod_{x \in \times, y \in \circ_{x}} d U_{x y} \int \prod_{x \in \times, \circ} d \bar{\psi}_{x} d \psi_{x} \exp \left[S[U]+\sum_{x \in \times, y \in \circ} \frac{\eta_{x y}}{2}\left(\bar{\psi}_{x}^{i, a} U_{x y}^{i j} \psi_{y}^{j, a}-\bar{\psi}_{y}^{j, a} U_{x y}^{\dagger j i} \psi_{x}^{i, a}\right)-\sum_{x \in \times, \circ} \bar{\psi}_{x}^{i, a} m^{a b} \psi_{x}^{i, b}\right]
$$


Here the link variable $U_{x y}^{i j}$ takes its value in the gauge group $G=\mathrm{U}(N), \mathrm{O}(N)$, or $\operatorname{Sp}(2 N)$. The Haar measure of $G$ is denoted by $d U$, and $S[U]=$ $g^{-2} \sum_{\langle x y z w\rangle} \operatorname{tr} U_{x y} U_{z y}^{\dagger} U_{z w} U_{x w}^{\dagger}+$ c.c. is the plaquette gauge action. The site variables $\psi_{x}^{i, a}$ and $\bar{\psi}_{x}^{i, a}$ are independent Grassmannian numbers. The color indices $i, j, \ldots$ run from 1 to $N$ for $G=\mathrm{U}(N), \mathrm{O}(N)$ or to $2 N$ for $G=\operatorname{Sp}(2 N)$, and the flavor indices $a, b, \ldots$ run from 1 to $n$. The phase is defined by $\eta_{x x \pm \hat{\mu}}=(-1)^{x^{1}+\cdots+x^{\mu-1}}$, and the quark mass matrix is by $m=\operatorname{diag}\left(m_{1}, \ldots, m_{n}\right)$.

As the fundamental representation of $\mathrm{O}(N)(\operatorname{Sp}(2 N))$ is real (pseudoreal), i.e. its symmetric (antisymmetric) product contains an invariant, the chiral symmetry group is extended to $\mathrm{U}(2 n)$ for these cases [5]. This can be made apparent by introducing $2 n$-flavored site variables

$$
\begin{aligned}
& \Psi_{y}^{i, A}=\left[\begin{array}{lll}
\psi_{y}^{i, a} & \bar{\psi}_{y}^{i, a}
\end{array}\right] \text { or }\left[\begin{array}{ll}
\psi_{y}^{i, a} & E^{i j} \bar{\psi}_{y}^{j, a}
\end{array}\right](y \in \circ), \\
& \bar{\Psi}_{x}^{i, A}=\left[\begin{array}{lll}
\bar{\psi}_{x}^{i, a} & \psi_{x}^{i, a}
\end{array}\right] \text { or }\left[\begin{array}{ll}
\bar{\psi}_{x}^{i, a} & E^{i j} \psi_{x}^{j, a}
\end{array}\right](x \in \times),
\end{aligned}
$$

$\left(A, B=1, \ldots, 2 n, E=i \sigma_{2} \otimes \mathbb{1}_{N}\right)$ for $G=\mathrm{O}(N)$ or $\mathrm{Sp}(2 N)$, resp., and writing the fermionic action as

$$
\begin{aligned}
& \sum_{x \in \times, y \in \circ_{x}} \frac{\eta_{x y}}{2} \bar{\Psi}_{x}^{i, A} O_{x y}^{i j} \Psi_{y}^{j, A}-\frac{1}{2} \sum_{y \in \circ} \Psi_{y}^{A, i} m^{A B} \Psi_{y}^{B, i}+\frac{1}{2} \sum_{x \in \times} \bar{\Psi}_{x}^{A, i} m^{A B} \bar{\Psi}_{x}^{B, i}\left(O_{x y} \in \mathrm{O}(N)\right), \\
& \sum_{x \in \times, y \in \circ_{x}} \frac{\eta_{x y}}{2} \bar{\Psi}_{x}^{i, A} S_{x y}^{i j} \Psi_{y}^{j, A}-\frac{1}{2} \sum_{y \in \circ} \Psi_{y}^{A, i} E^{i j} m^{A B} \Psi_{y}^{B, i}+\frac{1}{2} \sum_{x \in \times} \bar{\Psi}_{x}^{A, i} E^{i j} m^{A B} \bar{\Psi}_{x}^{B, i} \quad\left(S_{x y} \in \operatorname{Sp}(2 N)\right) .
\end{aligned}
$$

Extended mass matrices $m^{A B}$ in the above are defined by $m=\left(i \sigma_{2}\right.$ or $\left.\sigma_{1}\right) \otimes \operatorname{diag}\left(m_{1}, \ldots, m_{n}\right)$ for $G=\mathrm{O}(N)$ or $\operatorname{Sp}(2 N)$, respectively. The protected vector subgroup of this extended chiral group $\mathrm{U}(2 n)$ is read off from Eq.(3) to be $\operatorname{Sp}(2 n)$ for $G=\mathrm{O}(N)$, or $\mathrm{O}(2 n)$ for $G=\operatorname{Sp}(2 N)$.

Color-Flavor Transformation. Henceforth we concentrate on the strong coupling limit $g^{2} N \rightarrow \infty$. The theory is thus referred to as a non-Abelian random flux model.
In this case, $\eta_{x y}$ can be absorbed into the redefinition of the link variables. We can reexpress the partition function of this model in terms of an integration over flavor-singlet link variables into the one over color-singlet link variables, by Zirnbauer's color-flavor transformation. That for the $\mathrm{U}(N)$ link variable coupled to fermions reads [10,11, up to an irrelevant numerical factor,

$$
\int_{\mathrm{U}(N)} d U \exp \left(\bar{\psi}_{1}^{i, a} U^{i j} \psi_{0}^{j, a}-\bar{\psi}_{0}^{j, a} U^{\dagger j i} \psi_{1}^{i, a}\right)=\int \frac{\prod_{1 \leq a, b \leq n} d Z^{a b} d \bar{Z}^{a b}}{\operatorname{det}^{N+2 n}\left(1+Z^{\dagger} Z\right)} \exp \left(\bar{\psi}_{1}^{i, a} Z^{a b} \psi_{1}^{i, b}+\bar{\psi}_{0}^{i, a} Z^{\dagger a b} \psi_{0}^{i, b}\right) .
$$

The integration on the RHS is over complex $n \times n$ matrices, $\mathcal{N}=\mathrm{GL}(n, \mathbf{C})$. Although the case with the $\mathrm{O}(N)$ link variable coupled to fermions was not explicitly provided (see Ref. [11] for its bosonic counterpart), one can show that

$$
\int_{\mathrm{O}(N)} d O \exp \left(\bar{\Psi}^{i, A} O^{i j} \Psi^{j, A}\right)=\int_{Z=-Z^{T}} \frac{\prod_{1 \leq a<b \leq 2 n} d Z^{a b} d \bar{Z}^{a b}}{\operatorname{det}^{N / 2+2 n-1}\left(1+Z^{\dagger} Z\right)} \exp \left(\bar{\Psi}^{i, A} Z^{A B} \bar{\Psi}^{i, B}-\Psi^{i, A} Z^{\dagger A B} \Psi^{i, B}\right) .
$$

The integration on the RHS is over the set of complex $2 n \times 2 n$ antisymmetric matrices, which is diffeomorphic to $\mathcal{N}=\mathrm{GL}(2 n, \mathbf{C}) / \mathrm{Sp}(2 n, \mathbf{C})$. The case with the $\operatorname{Sp}(2 N)$ link variable coupled to fermions reads 10

$$
\int_{\mathrm{Sp}(2 N)} d S \exp \left(\bar{\Psi}^{i, A} S^{i j} \Psi^{j, A}\right)=\int_{Z=Z^{T}} \frac{\prod_{1 \leq a \leq b \leq 2 n} d Z^{a b} d \bar{Z}^{a b}}{\operatorname{det}^{N+2 n+1}\left(1+Z^{\dagger} Z\right)} \exp \left(\frac{1}{2} \bar{\Psi}^{i, A} E^{i j} Z^{A B} \bar{\Psi}^{j, B}-\frac{1}{2} \Psi^{i, A} E^{i j} Z^{\dagger A B} \Psi^{j, B}\right) .
$$

The integration on the RHS is over the set of complex $2 n \times 2 n$ symmetric matrices, which is diffeomorphic to $\mathcal{N}=\mathrm{GL}(2 n, \mathbf{C}) / \mathrm{O}(2 n, \mathbf{C})$. Applying these transformations to each link and then integrating over fermions, we obtain

$$
\mathcal{Z}(m)=\int_{\mathcal{N}} \prod_{x \in \times,} \frac{d Z_{x y} d \bar{Z}_{x y}}{\operatorname{det}^{N+2 n+\epsilon}\left(1+Z_{x y}^{\dagger} Z_{x y}\right)} \prod_{x \in \times} \operatorname{det}^{N}\left(\sum_{y \in \circ_{x}} Z_{x y}-2 m\right) \prod_{y \in \circ} \operatorname{det}^{N}\left(\sum_{x \in x_{y}} Z_{x y}^{\dagger}-2 m\right) .
$$

Here $\epsilon=0,-1,1$ for $G=\mathrm{U}(N), \mathrm{O}(N), \mathrm{Sp}(2 N)$, respectively, and we have redefined $N / 2 \rightarrow N$ and $m \rightarrow 2 m$ for the $\mathrm{O}(N)$ case. Pfaffians from fermionic integrations $\operatorname{Pf}(X)=\int d \Psi \exp (\Psi X \Psi)$ arising in the $\mathrm{O}(N)$ and $\operatorname{Sp}(2 N)$ cases are replaced by $\operatorname{det}^{1 / 2}(X)$. In spite of residing on a link, $Z_{x y}$ variables resemble the mass matrix that couples to a fermions bilinear on an identical site, thereby indicating the spontaneous chiral symmetry breaking.

Homogeneity of the Saddle Point. So far the manipulation applied to our non-Abelian random flux models was exact. We finally make an approximation, by taking the $N \rightarrow \infty$ limit. The saddle point equations read 


$$
Z_{x y}^{-1}=\sum_{\substack{x^{\prime} \in x_{y} \\ x^{\prime} \neq x}} Z_{x^{\prime} y}^{\dagger}-2 m,\left(Z_{x y}^{\dagger}\right)^{-1}=\sum_{\substack{y^{\prime} \in \in_{x} \\ y^{\prime} \neq y}} Z_{x y^{\prime}}-2 m
$$

$\left(x \in \times, y \in \circ_{x}\right)$ for $G=\mathrm{U}(N)$. Those for $G=\mathrm{O}(N)$ or $\operatorname{Sp}(2 N)$ are identical as the above, with $Z_{x y}$ constrained to be antisymmetric or symmetric.

Now we shall demonstrate that the unique solution to Eq.(6) at $m=0$ is given by

$$
Z_{x y}=(2 d-1)^{-1 / 2} U, U \in \mathrm{U}(n) \text { for }{ }^{\forall} x \in \times,{ }^{\forall} y \in \circ_{x},
$$

i.e. the vacuum configuration of the mesonic variable is homogeneous. In other two cases, we simply need to restrict $U$ to be an antisymmetric $(G=\mathrm{O}(N))$ or a symmetric $(G=\operatorname{Sp}(2 N))$ unitary matrix, which in turn can be parametrized by another unitary matrix $V \in \mathrm{U}(2 n)$ by the canonical projection $U=V J V^{T}\left(J=i \sigma_{2} \otimes \mathbb{1}_{n}\right)$ or $U=V V^{T}$, respectively.

The set of saddle point equations involving an even site $x$ read, after using abbreviations $Z_{p} \equiv Z_{x y_{p}}$ for $y_{1}, \ldots, y_{2 d} \in o_{x}$,

$$
\left(Z_{p}^{\dagger}\right)^{-1}=\sum_{q=1(q \neq p)}^{2 d} Z_{q}, \quad p=1, \ldots, 2 d(\geq 4) .
$$

Here $Z_{1}, \ldots, Z_{2 d} \in \mathrm{GL}(n, \mathbf{C})$. Eq.(8) clearly leads to

$$
\left(Z_{p}^{\dagger}\right)^{-1}+Z_{p}=\left(Z_{q}^{\dagger}\right)^{-1}+Z_{q}, \quad \forall p, \forall q=1, \ldots, 2 d .
$$

We make singular value decomposition of the matrices

$$
Z_{p}=U_{p} S_{p} V_{p}, \quad S_{p}=\operatorname{diag}\left(s_{1}^{(p)}, \ldots, s_{n}^{(p)}\right),
$$

where $U_{p}, V_{p} \in \mathrm{U}(n)$ and $s_{1}^{(p)}, \ldots, s_{n}^{(p)}>0$, and define $\sigma_{a}^{(p)}=1 / s_{a}^{(p)}+s_{a}^{(p)}$. Without loss of generality we choose $\left(U_{p}, V_{p}\right)$ so that $\sigma_{1}^{(p)} \geq \cdots \geq \sigma_{n}^{(p)}$. From Eq. (9)), we have

$$
\begin{aligned}
U_{p}\left(S_{p}^{-1}+S_{p}\right) V_{p} & =U_{q}\left(S_{q}^{-1}+S_{q}\right) V_{q} \\
\Rightarrow U_{q}^{\dagger} U_{p}\left(S_{p}^{-1}+S_{p}\right) V_{p} V_{q}^{\dagger} & =V_{q} V_{p}^{\dagger}\left(S_{p}^{-1}+S_{p}\right) U_{p}^{\dagger} U_{q} \\
& =S_{q}^{-1}+S_{q} \\
\Rightarrow U_{q}^{\dagger} U_{p}\left(S_{p}^{-1}+S_{p}\right)^{2} U_{p}^{\dagger} U_{q} & =\left(S_{q}^{-1}+S_{q}\right)^{2} .
\end{aligned}
$$

From the uniqueness of the eigenvalues, we find that the diagonal matrix $S_{p}^{-1}+S_{p}$ is independent of $p$, and thus is simply denoted as $S_{p}^{-1}+S_{p}=\operatorname{diag}\left(\sigma_{1}, \ldots, \sigma_{n}\right)$. Then Eq.(12) in a componentwise notation reads

$$
\left(U_{q}^{\dagger} U_{p}\right)^{a b}\left(\sigma_{b}^{2}-\sigma_{a}^{2}\right)=0, \quad a, b=1, \ldots, n .
$$

As $\sigma_{b}+\sigma_{a}$ is nonzero, we find

$$
\begin{aligned}
& \left(U_{q}^{\dagger} U_{p}\right)^{a b}\left(\sigma_{b}-\sigma_{a}\right)=0, \quad a, b=1, \ldots, n \\
\Leftrightarrow & U_{q}^{\dagger} U_{p}\left(S_{p}^{-1}+S_{p}\right)=\left(S_{p}^{-1}+S_{p}\right) U_{q}^{\dagger} U_{p} .
\end{aligned}
$$

Using Eq.(15) in Eq.(11), we obtain

$$
U_{q}^{\dagger} U_{p}=V_{q} V_{p}^{\dagger} \equiv W_{q p}
$$

Note that $W_{q p}^{\dagger}=W_{p q}$. In terms of $W_{q p}$, Eq.(8) reads

$$
S_{p}^{-1}=\sum_{q=1}^{2 d} W_{q p}^{\dagger} S_{q} W_{q p}, \quad p=1, \ldots, 2 d .
$$

Let us now suppose $\sigma_{1}=\cdots=\sigma_{r}>\sigma_{r+1} \geq \cdots \geq \sigma_{n}$ and consider possible two cases separately.

In the case $r=n$, we have

$$
S_{p}^{-1}+S_{p}=\sigma \mathbb{1}_{n}, \quad p=1, \ldots, 2 d .
$$

We rewrite the saddle point equations (8) as

$$
\sum_{r=1}^{2 d} W_{(r \neq p, q)}^{\dagger} S_{r} W_{r p}=S_{p}^{-1}-W_{q p}^{\dagger} S_{q} W_{q p},
$$

where $p, q=1, \ldots, 2 d$ and $p \neq q$. Since $W_{r p}^{\dagger} S_{r} W_{r p}$ are positive-definite, the LHS of Eq.(19) is positive-definite. Therefore $S_{p}^{-1}$ and $W_{q p}^{\dagger} S_{q} W_{q p}$ are ordered hermitian matrices [13] so that the largest and smallest diagonal elements (eigenvalues) of $S_{p}^{-1}$ are larger than the largest and smallest diagonal elements of $S_{q}$, respectively. Suppose the largest diagonal element of $S_{p}^{-1}$ is $s$. From Eq.(18), the diagonal elements of $S_{p}$ and $S_{q}$ are $s$ or $1 / s$. The largest diagonal element of $S_{q}$ is $1 / s$ and $s>1$, since it must be smaller than $s$. Therefore all the diagonal elements of $S_{q}$ are $1 / s$. Then the smallest diagonal element of $S_{p}^{-1}$ has to be $s$, because it must be larger than the smallest diagonal element of $S_{q}$. Consequently all the diagonal elements of $S_{p}^{-1}$ are $s$ and we conclude

$$
Z_{p}=U_{p} S_{p} V_{p}=U_{p} V_{p} S_{p}=U_{q} V_{q} S_{q}=U_{q} S_{q} V_{q}=Z_{q} .
$$

In the case $r<n$, we obtain from Eqs.(14) and (16),

$$
\left(W_{q p}\right)^{a b}=0 \text { for }\left\{\begin{array}{l}
a=1, \ldots, r, \quad b=r+1, \ldots, n \\
a=r+1, \ldots, n, \quad b=1, \ldots, r
\end{array}\right.
$$

for an arbitrary pair of indices $p$ and $q$. It means that the saddle point equation (17) is decomposed into those with smaller ranks $r$ and $n-r$. Thus we can inductively show $Z_{1}=\cdots=Z_{2 d}$, using the argument given in the previous case for each irreducible component.

Substituting $Z_{1}=\cdots=Z_{2 d}$ into Eq.(8), we obtain

$$
Z_{p}=(2 d-1)^{-1 / 2} U, \quad U \in \mathrm{U}(n), \quad \forall p=1, \ldots, 2 d .
$$

As this proof applies for each of even sites, or odd sites with $Z_{x y}$ substituted by $Z_{x y}^{\dagger}$, we finally establish Eq.(7).

Chiral Lagrangian. Next we take into account a small deviation from the chiral limit, as well as fluctuations of the mesonic field around the vacuum configuration that has been proven in the last section to be homogeneous.

The terms to be collected are of lowest nontrivial orders in masses and momenta, which are $O\left(m^{1}\right)$ and $O\left(\partial^{2}\right)$, 
respectively. In the case of $G=\mathrm{U}(N)$ [8], we split the mesonic variable into massive and Goldstone modes as

$$
Z_{x y}=(2 d-1)^{-1 / 2} P_{x y} U_{x y},
$$

where $P_{x y} \in \mathrm{GL}(n, \mathbf{C}) / \mathrm{U}(n)$ and $U_{x y} \in \mathrm{U}(n)$. In the case of $G=\mathrm{O}(N)$ or $\operatorname{Sp}(2 N)$, the mesonic variable is constrained to be antisymmetric or symmetric, respectively, so we must employ parameterizations

$$
\begin{aligned}
& Z_{x y}=(2 d-1)^{-1 / 2} P_{x y} V_{x y} J V_{x y}^{T} P_{x y}^{T} \quad(G=\mathrm{O}(N)), \\
& Z_{x y}=(2 d-1)^{-1 / 2} P_{x y} V_{x y} V_{x y}^{T} P_{x y}^{T} \quad(G=\mathrm{Sp}(2 N)),
\end{aligned}
$$

where $V_{x y}$ is the Goldstone mode over $\mathcal{M}=\mathrm{U}(2 n) /$ $\mathrm{Sp}(2 n)$ or $\mathrm{U}(2 n) / \mathrm{O}(2 n)$, resp., and $P_{x y}$ is the massive mode over $\mathcal{N} / \mathcal{M}$. Then we expand the Goldstone mode $U_{x y}=\exp \left(\partial_{x \vec{x}}\right) U_{x}$ up to quadratic order in the directional derivatives $\partial_{\vec{x} y}$, and Gaussian-integrate over the massive mode $P_{x y}=\exp \left(X_{x y}\right)$ by retaining up to quadratic order in $X_{x y}$. We reinstate the lattice spacing $a$ and employ dimensionful continuum notations

$$
m \rightarrow a M, \quad \sum_{y \in \times_{x}} \partial_{\overrightarrow{x y}}^{2} \rightarrow \frac{1}{2} a^{2} \nabla^{2}, \quad \sum_{x \in \times} \rightarrow \frac{1}{2} a^{-d} \int d^{d} x .
$$

Then the resulting effective partition function reads

$$
\begin{gathered}
\mathcal{Z}(M)=\int_{\mathcal{M}} \mathcal{D} V \exp \left[-\int d^{d} x\left(\frac{N}{8 d} a^{2-d} \operatorname{tr} \nabla U^{\dagger} \nabla U\right.\right. \\
\left.\left.-\frac{N \sqrt{2 d-1}}{d} a^{1-d} \operatorname{Re} \operatorname{tr} M U+C a^{2-d}\left(\operatorname{tr} U^{\dagger} \nabla U\right)^{2}\right)\right],
\end{gathered}
$$

where $U=V, V J V^{T}, V V^{T}$ for $G=\mathrm{U}(N), \mathrm{O}(N)$, $\operatorname{Sp}(2 N)$, respectively, and $\mathcal{D} V=\prod_{x \in \mathrm{x}} d V_{x}$. The coefficient $C$ of the singlet $\left(\eta^{\prime}\right)$ kinetic term is a constant of $O(1)$ depending on $d$ and $G$ [8], but we suppress its explicit form as $\eta^{\prime}$ should decouple from the Goldstone sector if the $\mathrm{U}(1)_{A}$ anomaly were properly taken into account. Consequently the pion decay constant squared and the chiral condensate (the coefficients of the first and the second terms) are derived from the first principle.

Conclusion. We have rigorously derived three classes of low-energy chiral Lagrangians from microscopic theories: non-Abelian random flux models, or lattice gauge theories with staggered fermions in the strong coupling limit. These nonlinear $\sigma$ models have symmetric spaces $\mathcal{M}=\mathrm{U}(n), \mathrm{U}(2 n) / \mathrm{Sp}(2 n), \mathrm{U}(2 n) / \mathrm{O}(2 n)$ as their target manifolds, depending on the complexity, reality, and pseudoreality of the defining representations of the gauge groups $G=\mathrm{U}(N), \mathrm{O}(N), \mathrm{Sp}(2 N)$, respectively. These results are anticipated from Vafa-Witten argument [12] or equivalently from the anti-unitary symmetries of the staggered Dirac operators in Eq.(1), whose matrix elements are trivially complex, real, and quaternion real for these three gauge groups. For the last two cases of the gauge groups, the anti-unitary symmetries of lattice Dirac operators are interchanged from those of the continuum Dirac operators, due to the absence of the charge conjugation matrix acting on spinor indices, that squares to -1 14. The puzzle of if and how the crossover between different classes of effective theories could occur, originally posed for staggered fermions in the adjoint of $\mathrm{SU}(2)$ (= fundamental of $\mathrm{O}(3)$ ) and in the fundamental of $\mathrm{SU}(2)$ ( $\approx$ fundamental of $\mathrm{Sp}(2)$ ), remains unsolved. Although the Wess-Zumino term in $d=2$ dimensions is of the same order as the kinetic term, our models involving staggered fermions do not yield it.

Verbaarschot [15] has conjectured that the three patterns of chiral symmetry breaking induce the spectral fluctuation of the Dirac operators to obey three universality classes of chiral random matrices [16]. If our parameters are restricted to the 'ergodic' domain where the size of the lattice is much smaller than the Compton length of pseudo-Goldstone bosons, the chiral Lagrangians are dominated by their zero modes, i.e. become finite-dimensional integrals involving only the mass terms in Eq.(24) [17. As these three 'finite-volume' partition functions are known [16, 18] to be equivalent to chiral random matrix ensembles at $\beta=2,1,4$ in the large matrixdimension limit, we have also proven his conjecture.

* $\quad$ Supported by JSPS and by Grant-in-Aid No. 411044 from the Ministry of Education, Science and Culture, Japan.

[1] K.B. Efetov, Adv. Phys. 32, 53 (1983).

[2] S. Weinberg, Phys. Rev. Lett. 18, 188 (1967).

[3] C. Vafa and E. Witten, Nucl. Phys. B234, 173 (1984).

[4] G. 't Hooft, in Recent Developments in Gauge Theories, eds. G. 't Hooft et al., Plenum Press (New York, 1980).

[5] M.E. Peskin, Nucl. Phys. B175, 197 (1980).

[6] H. Kluberg-Stern et al., Nucl. Phys. B190, 504 (1981); N. Kawamoto and J. Smit, Nucl. Phys. B192, 100 (1981).

[7] T. Jolicoeur et al., Nucl. Phys. B235, 455 (1984); I. Ichinose, Nucl. Phys. B249, 715 (1985).

[8] A. Altland and B.D. Simons, Nucl. Phys. B562, 445 (1999).

[9] E. Brézin and D.J. Gross, Phys. Lett. B97, 120 (1980).

[10] M.R. Zirnbauer, J. Math. Phys. 38, 2007 (1997).

[11] M.R. Zirnbauer, chao-dyn/9810016.

[12] J.B. Kogut, M.A. Stephanov, and D. Toublan, Phys. Lett. B464, 183 (1999).

[13] M.L. Mehta, Matrix Theory, Les Editions de Physique (Paris, 1989).

[14] M.A. Halasz and J.J.M. Verbaarschot, Phys. Rev. Lett. 74, 3920 (1995).

[15] J.J.M. Verbaarschot, Phys. Rev. Lett. 72, 2531 (1994).

[16] E.V. Shuryak and J.J.M. Verbaarschot, Nucl. Phys. A560, 306 (1993).

[17] H. Leutwyler and A. Smilga, Phys. Rev. D46, 5607 (1992); A. Smilga and J.J.M. Verbaarschot, Phys. Rev. D51, 829 (1995).

[18] M.A. Halasz and J.J.M. Verbaarschot, Phys. Rev. D52, 2563 (1995). 